\title{
WELL-POSEDNESS OF THE ITERATIVE BOLTZMANN INVERSION
}

\author{
MARTIN HANKE*
}

\begin{abstract}
The iterative Boltzmann inversion is an iterative scheme to determine an effective pair potential for an ensemble of identical particles in thermal equilibrium from the corresponding radial distribution function. Although the method is reported to work reasonably well in practice, it still lacks a rigorous convergence analysis. In this paper we provide some first steps towards such an analysis, and we show under quite general assumptions that the algorithm is well-defined in a neighborhood of the true pair potential, assuming that such a potential exists.

On our way we establish important properties of the cavity distribution function and provide a proof of a statement formulated by Groeneveld concerning the rate of decay at infinity of the Ursell function associated with a Lennard-Jones type potential.
\end{abstract}

Key words. Statistical mechanics, cluster expansion, grand canonical ensemble, radial distribution function, cavity distribution function, Fréchet derivative

AMS subject classifications. $82 \mathrm{~B} 21,82 \mathrm{~B} 80$

Last modified. February 22, 2018

1. Introduction. Numerical simulations of complex materials in physical chemistry are so time-consuming, even with today's computing power at hand, that it is necessary to implement subprocesses on a meso-scale by means of "coarse-graining" the atomistic structure of (parts of) the associated molecules. The coarse-grained "beads" are simulated by using effective potentials for their interactions. These effective potentials have to be determined a priori, and this is often done so as to match some given structural data.

Here we consider the case where this structural information consists of measurements of the so-called radial distribution function $g^{\dagger}$ of the beads; see (3.2) for a formal definition of this function. The effective potential $u^{\dagger}$ is then chosen in such a way that

$$
F\left(u^{\dagger}\right)=g^{\dagger}
$$

where $F$ is the function which maps a potential $u$ (out of a predetermined family of suitable functions) onto the corresponding radial distribution function of the associated grand canonical ensemble under well-defined physical conditions. The question of existence and uniqueness of a solution $u^{\dagger}$ of (1.1) for a given $g^{\dagger}$ is referred to as the inverse Henderson problem, because Henderson [8] was the first to investigate the identifiability problem associated with (1.1), i.e., whether the radial distribution function is enough data to uniquely recover the underlying pair potential; see Kuna, Lebowitz, and Speer [10] for a more rigorous mathematical treatment of the uniqueness problem.

A popular method for solving numerically the inverse Henderson problem is the iterative Boltzmann inversion (IBI) suggested by Soper [14]. This method, which is available in pertinent public domain software like $\operatorname{VOTCA}^{\dagger}[11]$ starts from an initial guess $u_{0}$ and determines recursively an iterative sequence $\left(u_{k}\right)_{k \geq 0}$ of approximate

\footnotetext{
*Institut für Mathematik, Johannes Gutenberg-Universität Mainz, 55099 Mainz, Germany (hanke@math.uni-mainz.de). The research leading to this work has been done within the Collaborative Research Center TRR 146; corresponding funding by the DFG is gratefully acknowledged.

${ }^{\dagger}$ http://www.votca.org
} 
solutions of (1.1) via

$$
u_{k+1}=u_{k}+\gamma \log \frac{F\left(u_{k}\right)}{g^{\dagger}}, \quad k=0,1,2, \ldots
$$

Here $\gamma>0$ is a relaxation parameter that is usually chosen to be

$$
\gamma=1 / \beta
$$

where $\beta>0$ is the inverse temperature.

A mathematical analysis of the IBI method is still lacking although the method seems to be fairly robust. In his original paper [14] Soper provided a heuristic argument why IBI might be expected to converge, however, there is little hope to turn this argument into a rigorous proof.

From a mathematical point of view a possible framework for studying (1.2) is fixed point iteration theory, where

$$
\Phi(u)=u+\gamma \log \frac{F(u)}{g^{\dagger}}
$$

is the corresponding fixed point operator. This is the point of view taken in this paper. Note, however, that currently we are not yet able to prove existence of a fixed point of $\Phi$ under reasonable assumptions on $g^{\dagger}$ and the set of admissible pair potentials, although this is evidently a necessary requirement for convergence of (1.2); the only result in this direction that we are aware of has been provided by Koralov [9].

Instead we stipulate that a solution $u^{\dagger}$ of (1.1) exists, in which case we show that the first few iterates of (1.2) are well-defined when the initial guess $u_{0}$ is chosen from a suitable neighborhood of $u^{\dagger}$. Additional assumptions that we impose are (i) that $u^{\dagger}$ is a Lennard-Jones type potential, and (ii) that the grand canonical ensemble is in the so-called gas phase; see below.

The outline of this paper is as follows. In the following section we specify our requirements on the family of admissible pair potentials. We then review in Section 3 the necessary background concerning the associated grand canonical ensemble and its thermodynamical limit, and investigate in more detail the so-called cavity distribution function. Section 4 contains an auxiliary result on autoconvolution products of a certain class of functions which include the Mayer function. This will be applied in Section 5 to discuss the rate of decay of the Ursell function for large radii and to improve upon our earlier results in [5] on the derivative of the Ursell function with respect to the pair potential. This provides the main ingredients of our analysis of the IBI method in Section 6.

We mention that although we treat IBI in the context of a grand canonical ensemble, it is possible to extend this analysis to a canonical ensemble, which is the more usual setting of numerical simulations in practice.

2. Setting. We start by considering an ensemble of identical classical particles in thermal equilibrium within a bounded cubical box $\Lambda \subset \mathbb{R}^{3}$ centered at the origin. We assume that the interaction of the particles can be described by a pair potential $u=u(r)$, which only depends on the distance $r>0$ of the interacting particles, and that this potential satisfies

$$
\begin{array}{cl}
|u(r)| \leq C r^{-\alpha}, & r \geq r_{0}, \\
u(r) \geq c r^{-\alpha}, & r \leq r_{0},
\end{array}
$$


for some $\alpha>3, r_{0}>0$, and parameters $c, C$ satisfying $C_{0}>C>c>c_{0}>0$; here, $\alpha$, $r_{0}, c_{0}$, and $C_{0}$ are fixed constants, and we denote by $\mathscr{U}=\mathscr{U}\left(\alpha, r_{0}, c_{0}, C_{0}\right)$ the family of potentials $u$ satisfying (2.1). Following Ruelle [13] potentials $u \in \mathscr{U}$ are called Lennard-Jones type pair potentials.

Under this assumption it is known (cf. Fischer and Ruelle [1]) that there exists $B>0$ such that

$$
U_{N}\left(\boldsymbol{R}_{N}\right):=\sum_{1 \leq i<j \leq N} u\left(\left|R_{i}-R_{j}\right|\right) \geq-B N
$$

for every configuration of $N$ particles in free space and every $N \in \mathbb{N}$; here we denote by $R_{i} \in \mathbb{R}^{3}$ the coordinates of the $i$ th particle, and by $\boldsymbol{R}_{N}=\left(R_{1}, \ldots, R_{N}\right) \in\left(\mathbb{R}^{3}\right)^{N}$ the configuration of the first $N$ particles. It follows that for every $N \in \mathbb{N}$ and $\boldsymbol{R}_{N} \in\left(\mathbb{R}^{3}\right)^{N}$ there is an index $i^{*}\left(\boldsymbol{R}_{N}\right)$ such that

$$
\sum_{\substack{i=1 \\ i \neq i^{*}}}^{N} u\left(\left|R_{i}-R_{i^{*}}\right|\right) \geq-2 B .
$$

Associated with $u \in \mathscr{U}$ and the inverse temperature $\beta>0$ is the Mayer function

$$
f(R)=e^{-\beta u(|R|)}-1,
$$

which is considered to be -1 at the origin $R=0$. Because of (2.1) the Mayer function is absolutely integrable, i.e., there exists $c_{\beta}>0$ such that

$$
\int_{\mathbb{R}^{3}}|f(R)| \mathrm{d} R<c_{\beta} .
$$

By virtue of (2.2) and (2.5) every Lennard-Jones type potential is stable and regular in the sense of [13].

As worked out in the proof of [4, Proposition 2.1], for every $u \in \mathscr{U}$ the same constants $B$ and $c_{\beta}$ can be used in (2.2) and (2.5), respectively, and also the indices $i^{*}$ in (2.3) can be chosen independent of $u \in \mathscr{U}$.

Associated with the constant $\alpha$ in (2.1) is the weight function

$$
\varrho(r)=\left(1+r^{2}\right)^{\alpha / 2}, \quad r \geq 0 .
$$

For every $u \in \mathscr{U}$ we can use this weight function to define a corresponding Banach space $\mathscr{V}_{u}$ of perturbations, consisting of all functions $v$ for which the associated norm

$$
\|v\|_{\mathscr{V}_{u}}=\max \left\{\|v / u\|_{\left(0, r_{0}\right]},\|\varrho v\|_{\left[r_{0}, \infty\right)}\right\}
$$

is finite. Clearly, for every $u \in \mathscr{U}$ there exists $\delta_{0}=\delta_{0}(u) \in(0,1)$ sufficiently small such that $u+v \in \mathscr{U}$ for every $v \in \mathscr{V}_{u}$ with $\|v\|_{\mathscr{V}_{u}} \leq \delta_{0}$. Later, compare (5.6) and (5.7), we will reduce the size of $\delta_{0}(u)$ somewhat further to ensure additional properties of $u+v$ for all $v \in \mathscr{V}_{u}$ with $\|v\|_{\mathscr{V}_{u}} \leq \delta_{0}$.

3. The grand canonical ensemble. Let $u \in \mathscr{U}$ be the pair potential that determines the interaction of the particles. In the grand canonical ensemble the number of particles and their coordinates within $\Lambda$ are random variables, and the probability of observing an ensemble with exactly $N$ particles in an infinitesimal volume $\mathrm{d} \boldsymbol{R}_{N}$ at the coordinates $\boldsymbol{R}_{N} \in \Lambda^{N}$ (up to permutations) is given by

$$
\frac{1}{\Xi_{\Lambda}(z)} \frac{z^{N}}{N !} e^{-\beta U_{N}\left(\boldsymbol{R}_{N}\right)} \mathrm{d} \boldsymbol{R}_{N}
$$


where $z>0$ is the so-called activity, $U_{N}\left(\boldsymbol{R}_{N}\right)$ is defined in (2.2), and

$$
\Xi_{\Lambda}(z)=\sum_{N=0}^{\infty} \frac{z^{N}}{N !} \int_{\Lambda^{N}} e^{-\beta U_{N}\left(\boldsymbol{R}_{N}\right)} \mathrm{d} \boldsymbol{R}_{N}
$$

is the grand canonical partition function. We consider this grand canonical ensemble under specified physical conditions, i.e., we assume that the activity $z$ and the inverse temperature $\beta$ are given (and fixed), and that they satisfy the inequality

$$
0<z<\frac{1}{c_{\beta} e^{2 \beta B+1}}
$$

where $c_{\beta}$ and $B$ are the constants in (2.5) and (2.2), respectively. This regime is known as the gas phase of the ensemble, cf. [13].

For $m \in \mathbb{N}$ and $\boldsymbol{R}_{m} \in \Lambda^{m}$ the $m$-particle distribution function is given by

$$
\rho_{\Lambda}^{(m)}\left(\boldsymbol{R}_{m}\right)=\frac{1}{\Xi_{\Lambda}(z)} \sum_{N=m}^{\infty} \frac{z^{N}}{(N-m) !} \int_{\Lambda^{N-m}} e^{-\beta U_{N}\left(\boldsymbol{R}_{N}\right)} \mathrm{d} \boldsymbol{R}_{m, N},
$$

where $\boldsymbol{R}_{m, N}=\left(R_{m+1}, \ldots, R_{N}\right) ; \rho_{\Lambda}^{(m)}$ determines the probability distribution for snap shots with $m$ particles (up to permutations) at coordinates $R_{1}, \ldots, R_{m} \in \Lambda . \ddagger$ The grand canonical partition function $\Xi_{\Lambda}$ can be seen to be an entire function of $z \in \mathbb{C}$, which is free of zeros for

$$
z \in \mathcal{Z}=\left\{z \in \mathbb{C}:|z|<\frac{1}{c_{\beta} e^{2 \beta B+1}}\right\}
$$

compare [13, Theorem 4.2.3], and similarly, the $m$-particle distribution functions all are analytic functions of $z \in \mathcal{Z}$. On the other hand, the $m$-particle distribution functions may encounter singularities for positive values of $z$ outside the interval (3.1); those are understood to correspond to physical phase transitions.

As shown in [13] the $m$-particle distribution function has a well-defined thermodynamical limit, i.e., $\rho_{\Lambda}^{(m)}$ converges to some $\rho^{(m)} \in L^{\infty}\left(\left(\mathbb{R}^{3}\right)^{m}\right)$ as $|\Lambda| \rightarrow \infty$, uniformly on every compact subset of $\left(\mathbb{R}^{3}\right)^{m}$ and for activities $z$ from every compact subset of $\mathcal{Z}$, this being true for every $m \in \mathbb{N}$; here, $|\Lambda|$ denotes the volume of the box. In particular, for $m=1$, the thermodynamical limit

$$
\rho^{(1)}(R)=\rho_{0} \in \mathbb{R}_{0}^{+}
$$

is independent of $R \in \mathbb{R}^{3}$ and provides the counting density of the ensemble; for $m=2, \rho^{(2)}\left(R_{1}, R_{2}\right)$ only depends on the distance $r=\left|R_{1}-R_{2}\right| \geq 0$. Given these two functions the radial distribution function, referred to in the introduction, is defined to be

$$
g(r)=\frac{1}{\rho_{0}^{2}} \rho^{(2)}(R, 0), \quad|R|=r \geq 0 .
$$

For $m \in \mathbb{N}_{0}$ and $z \in \mathcal{Z}$ the function $\rho^{(m)}$ is Fréchet differentiable with respect to $u$, i.e., with respect to perturbations $v \in \mathscr{V}_{u}$ of $u$, cf. [4]. The derivative is a bounded linear operator $\partial \rho^{(m)} \in \mathscr{L}\left(\mathscr{V}_{u}, L^{\infty}\left(\left(\mathbb{R}^{3}\right)^{m}\right)\right)$. A similar result (see [4, Remark 3.4] for

\footnotetext{
${ }^{\ddagger}$ If $R_{i}=R_{j}$ for different indices $i, j \in\{1, \ldots, m\}$ then $\rho_{\Lambda}^{(m)}\left(\boldsymbol{R}_{m}\right)$ is set to be zero.
} 
details) applies to certain weighted copies of the particle distribution functions, which include the cavity distribution function (cf. Hansen and McDonald [6])

$$
y(r)=e^{\beta u(r)} g(r), \quad r>0,
$$

as a special case; the following result elaborates on this.

Proposition 3.1. For $u \in \mathscr{U}$ and $z \in \mathcal{Z}$ the cavity distribution function $y$ of (3.3) is a bounded function of $r>0$, which is analytic with respect to $z \in \mathcal{Z}$ and uniformly bounded on every compact subset of $\mathcal{Z}$. Moreover, $y$ is Fréchet differentiable with respect to $u$ with derivative $\partial y \in \mathscr{L}\left(\mathscr{V}_{u}, L^{\infty}\left(\mathbb{R}^{+}\right)\right)$. For

$$
0<z \leq \bar{z}<\frac{1}{1+e} \frac{1}{c_{\beta} e^{2 \beta B+1}}
$$

the cavity distribution function is strictly positive, i.e., there exists $c>0$ (depending only on $\bar{z}$ ) such that

$$
y(r) \geq c \frac{z^{2}}{\rho_{0}^{2}}, \quad r>0,
$$

for all $u \in \mathscr{U}$.

Proof. The function

$$
\sigma^{(2)}(R, 0)=\rho_{0}^{2} y(|R|)
$$

is the second entry of the semi-infinite vector $\boldsymbol{\sigma}=\boldsymbol{\sigma}_{\mathbb{R}^{3}}$ considered in [4, Remark 3.4]. There it is shown that $\boldsymbol{\sigma}$ satisfies a system

$$
(I-z B) \sigma=z e_{1}, \quad B=K D,
$$

of Kirkwood-Salsburg integral equations, and that $\boldsymbol{\sigma}$ has certain differentiability properties. These properties readily imply differentiability of $y$ with respect to $z$ and $u$ as stated above.

In (3.6) we have adopted notation of [4]: $K$ is a semi-infinite matrix of integral operators, $D$ a diagonal multiplication operator, and $I$ the corresponding identity operator; $\boldsymbol{e}_{1}$ is a vector of constant functions, its first entry being identically one, and all other entries being zero. To establish the lower bound (3.5) for the specific real interval of activity parameters $z$ given in (3.4), we first note that $I-z B$ can be developed into a Neumann series, and hence we can rewrite (3.6) in the form

$$
\boldsymbol{\sigma}=z \boldsymbol{e}_{\mathbf{1}}+z^{2} B \boldsymbol{e}_{\mathbf{1}}+\boldsymbol{h},
$$

where

$$
\boldsymbol{h}=\left(h^{(m)}\right)_{m}=z^{3}(I-z B)^{-1} B K D \boldsymbol{e}_{\mathbf{1}}=z^{3}(I-z B)^{-1} B K \boldsymbol{e}_{\mathbf{1}},
$$

because the $(1,1)$-entry of $D$ is an identity operator. Looking at the second entry of the vector identity (3.7) we conclude that

$$
\sigma^{(2)}(R, 0)-z^{2}=h^{(2)}(R, 0),
$$

because the second entry $b_{21}$ of $B \boldsymbol{e}_{1}$ is again a constant, i.e., $b_{21}=1$; compare [4]. For $z \in \mathcal{Z}$ the right-hand side of (3.8) can be bounded as in [4], which gives

$$
\left\|h^{(2)}\right\|_{L^{\infty}\left(\left(\mathbb{R}^{3}\right)^{2}\right)} \leq z^{3} e \frac{c_{\beta} e^{2 \beta B+1}}{1-z c_{\beta} e^{2 \beta B+1}} .
$$


Therefore there exists $c>0$ such that for every $R \in \mathbb{R}^{3}$ with $|R|=r>0$ there holds

$$
y(r)=\frac{\sigma^{(2)}(R, 0)}{\rho_{0}^{2}} \geq \frac{1}{\rho_{0}^{2}} z^{2}\left(1-z e \frac{c_{\beta} e^{2 \beta B+1}}{1-z c_{\beta} e^{2 \beta B+1}}\right) \geq c \frac{z^{2}}{\rho_{0}^{2}},
$$

provided $0<z \leq \bar{z}$.

4. An auxiliary inequality for autoconvolution products. Before we continue we define the Banach space $L_{\varrho}^{\infty}\left(\mathbb{R}^{3}\right)$ of functions $v \in L^{\infty}\left(\mathbb{R}^{3}\right)$ with finite norm

$$
\|w\|_{L_{\varrho}^{\infty}\left(\mathbb{R}^{3}\right)}=\sup _{R \in \mathbb{R}^{3}} \varrho(|R|)|w(R)|,
$$

where $\varrho$ is as in (2.6). We mention that the Mayer $f$-function defined in (2.4) belongs to this space by virtue of $(2.1)$. Note that $L_{o}^{\infty}\left(\mathbb{R}^{3}\right)$ is continuously embedded in $L^{1}\left(\mathbb{R}^{3}\right)$ and $L^{\infty}\left(\mathbb{R}^{3}\right)$, because the parameter $\alpha$ in (2.1) is assumed to satisfy $\alpha>3$. It readily follows that the convolution $w * w^{\prime}$ of two functions $w, w^{\prime} \in L_{\varrho}^{\infty}\left(\mathbb{R}^{3}\right)$ is an absolutely integrable function. In fact, we show next that the result belongs to $L_{\varrho}^{\infty}\left(\mathbb{R}^{3}\right)$ again.

Proposition 4.1. Let $w, w^{\prime} \in L_{\varrho}^{\infty}\left(\mathbb{R}^{3}\right)$. Then $w * w^{\prime} \in L_{\varrho}^{\infty}\left(\mathbb{R}^{3}\right)$ with

$$
\left\|w * w^{\prime}\right\|_{L_{\varrho}^{\infty}\left(\mathbb{R}^{3}\right)} \leq c_{\varrho} 2^{\alpha+1}\|w\|_{L_{\varrho}^{\infty}\left(\mathbb{R}^{3}\right)}\left\|w^{\prime}\right\|_{L_{\varrho}^{\infty}\left(\mathbb{R}^{3}\right)},
$$

where $c_{\varrho}$ is the embedding constant for the embedding of $L_{\varrho}^{\infty}\left(\mathbb{R}^{3}\right)$ into $L^{1}\left(\mathbb{R}^{3}\right)$.

Proof. For $R \in \mathbb{R}^{3}$ and $0<\varepsilon<1$ we consider the ball $\mathcal{B}_{\varepsilon|R|}(R) \subset \mathbb{R}^{3}$ of radius $\varepsilon|R|$ around $R$. Depending on whether $R^{\prime}$ is inside or outside this ball, there holds

$$
\begin{aligned}
1+\left|R^{\prime}\right|^{2} & \geq(1-\varepsilon)^{2}\left(1+|R|^{2}\right), & & R^{\prime} \in \mathcal{B}_{\varepsilon|R|}(R), \\
1+\left|R^{\prime}-R\right|^{2} & \geq \varepsilon^{2}\left(1+|R|^{2}\right), & & R^{\prime} \in \mathbb{R}^{3} \backslash \mathcal{B}_{\varepsilon|R|}(R) .
\end{aligned}
$$

Using (4.3a) it follows for every $R \in \mathbb{R}^{3}$ that

$$
\begin{aligned}
& \varrho(|R|)\left|\int_{\mathcal{B}_{\varepsilon|R|}(R)} w\left(R-R^{\prime}\right) w^{\prime}\left(R^{\prime}\right) \mathrm{d} R^{\prime}\right| \\
& \leq \int_{\mathcal{B}_{\varepsilon|R|}(R)} \frac{\varrho(|R|)}{\varrho\left(\left|R^{\prime}\right|\right)}\left|w\left(R-R^{\prime}\right)\right| \varrho\left(\left|R^{\prime}\right|\right)\left|w^{\prime}\left(R^{\prime}\right)\right| \mathrm{d} R^{\prime} \\
& \leq \frac{1}{(1-\varepsilon)^{\alpha}}\left\|w^{\prime}\right\|_{L_{\varrho}^{\infty}\left(\mathbb{R}^{3}\right)} \int_{\mathcal{B}_{\varepsilon|R|}(R)}\left|w\left(R-R^{\prime}\right)\right| \mathrm{d} R^{\prime} \\
& \leq \frac{1}{(1-\varepsilon)^{\alpha}}\|w\|_{L^{1}\left(\mathbb{R}^{3}\right)}\left\|w^{\prime}\right\|_{L_{\varrho}^{\infty}\left(\mathbb{R}^{3}\right)},
\end{aligned}
$$

while (4.3b) implies that

$$
\begin{aligned}
& \varrho(|R|)\left|\int_{\mathbb{R}^{3} \backslash \mathcal{B}_{\varepsilon|R|}(R)} w\left(R-R^{\prime}\right) w^{\prime}\left(R^{\prime}\right) \mathrm{d} R^{\prime}\right| \\
& \leq \int_{\mathbb{R}^{3} \backslash \mathcal{B}_{\varepsilon|R|}(R)} \frac{\varrho(|R|)}{\varrho\left(\left|R-R^{\prime}\right|\right)} \varrho\left(\left|R-R^{\prime}\right|\right)\left|w\left(R-R^{\prime}\right)\right|\left|w^{\prime}\left(R^{\prime}\right)\right| \mathrm{d} R^{\prime} \\
& \leq \frac{1}{\varepsilon^{\alpha}}\|w\|_{L_{\varrho}^{\infty}\left(\mathbb{R}^{3}\right)} \int_{\mathbb{R}^{3} \backslash \mathcal{B}_{\varepsilon|R|}(R)}\left|w^{\prime}\left(R^{\prime}\right)\right| \mathrm{d} R^{\prime} \leq \frac{1}{\varepsilon^{\alpha}}\|w\|_{L_{\varrho}^{\infty}\left(\mathbb{R}^{3}\right)}\left\|w^{\prime}\right\|_{L^{1}\left(\mathbb{R}^{3}\right)} .
\end{aligned}
$$


Adding these two inequalities we thus conclude that

$$
\begin{aligned}
\left\|w * w^{\prime}\right\|_{L_{\varrho}^{\infty}\left(\mathbb{R}^{3}\right)} & \leq \frac{1}{(1-\varepsilon)^{\alpha}}\|w\|_{L^{1}\left(\mathbb{R}^{3}\right)}\left\|w^{\prime}\right\|_{L_{\varrho}^{\infty}\left(\mathbb{R}^{3}\right)}+\frac{1}{\varepsilon^{\alpha}}\|w\|_{L_{\varrho}^{\infty}\left(\mathbb{R}^{3}\right)}\left\|w^{\prime}\right\|_{L^{1}\left(\mathbb{R}^{3}\right)} \\
& \leq\left(\frac{1}{(1-\varepsilon)^{\alpha}}+\frac{1}{\varepsilon^{\alpha}}\right) c_{\varrho}\|w\|_{L_{\varrho}^{\infty}\left(\mathbb{R}^{3}\right)}\left\|w^{\prime}\right\|_{L_{\varrho}^{\infty}\left(\mathbb{R}^{3}\right)},
\end{aligned}
$$

where $c_{\varrho}$ is the embedding constant for the embedding $L_{\varrho}^{\infty}\left(\mathbb{R}^{3}\right) \subset L^{1}\left(\mathbb{R}^{3}\right)$. By choosing $\varepsilon=1 / 2$ we finally obtain (4.2).

Proposition 4.1 implies that we can rescale the norm of $L_{\varrho}^{\infty}\left(\mathbb{R}^{3}\right)$ to make $L_{\varrho}^{\infty}\left(\mathbb{R}^{3}\right)$ a commutative Banach algebra with respect to convolution.

For $w \in L_{\varrho}^{\infty}\left(\mathbb{R}^{3}\right)$ and $n \in \mathbb{N}$ let $W_{n}$ be the $n$-fold autoconvolution of $w$, i.e.,

$$
W_{1}=w, \quad W_{n+1}=w * W_{n}, \quad n \geq 1 .
$$

By virtue of Proposition 4.1 each $W_{n}$ belongs to $L_{\varrho}^{\infty}\left(\mathbb{R}^{3}\right)$, and there holds

$$
\left\|W_{n}\right\|_{L^{1}\left(\mathbb{R}^{3}\right)} \leq\|w\|_{L^{1}\left(\mathbb{R}^{3}\right)}^{n}, \quad n \geq 1 .
$$

Proposition 4.2. Assume that $w \in L_{\varrho}^{\infty}\left(\mathbb{R}^{3}\right)$ satisfies

$$
\|w\|_{L^{1}\left(\mathbb{R}^{3}\right)}=q<1,
$$

and let $\bar{q} \in(q, 1)$. Then the autoconvolution products $W_{n}$ defined in (4.5) satisfy

$$
\left\|W_{n}\right\|_{L_{\varrho}^{\infty}\left(\mathbb{R}^{3}\right)} \leq C_{*} \bar{q}^{n}\|w\|_{L_{\varrho}^{\infty}\left(\mathbb{R}^{3}\right)}, \quad n \in \mathbb{N},
$$

for some constant $C_{*}>0$ depending only on $\alpha, q$, and $\bar{q}$.

Proof. We are going to prove by induction the inequality

$$
\left\|W_{n}\right\|_{L_{\varrho}^{\infty}\left(\mathbb{R}^{3}\right)} \leq \frac{1}{\varepsilon^{\alpha}}\|w\|_{L_{\varrho}^{\infty}\left(\mathbb{R}^{3}\right)} \frac{1-(q / \bar{q})^{n}}{1-q / \bar{q}} \bar{q}^{n-1},
$$

where we let

$$
\varepsilon=1-(q / \bar{q})^{1 / \alpha},
$$

which is a positive number; this readily implies (4.8). The induction base $n=1$ of (4.9) is obviously correct because $\varepsilon<1$ according to (4.10). For the induction step from $n$ to $n+1, n \geq 1$, we apply inequality (4.4) from the proof of Proposition 4.1 with $w^{\prime}=W_{n}$ and $\varepsilon$ of (4.10) to obtain

$$
\left\|W_{n+1}\right\|_{L_{\varrho}^{\infty}\left(\mathbb{R}^{3}\right)} \leq \bar{q}\left\|W_{n}\right\|_{L_{\varrho}^{\infty}\left(\mathbb{R}^{3}\right)}+\frac{1}{\varepsilon^{\alpha}}\|w\|_{L_{\varrho}^{\infty}\left(\mathbb{R}^{3}\right)}\left\|W_{n}\right\|_{L^{1}\left(\mathbb{R}^{3}\right)} .
$$

Inserting (4.6), (4.7), and the induction hypothesis (4.9) this yields

$$
\left\|W_{n+1}\right\|_{L_{\varrho}^{\infty}\left(\mathbb{R}^{3}\right)} \leq \frac{1}{\varepsilon^{\alpha}}\|w\|_{L_{\varrho}^{\infty}\left(\mathbb{R}^{3}\right)}\left(\frac{1-(q / \bar{q})^{n}}{1-q / \bar{q}}+(q / \bar{q})^{n}\right) \bar{q}^{n},
$$

which coincides with the bound (4.9) for the norm of $W_{n+1}$. $\square$

Corollary 4.3. Under the assumptions of Proposition 4.2 the infinite series

$$
W_{\Sigma}=\sum_{n=1}^{\infty} W_{n}
$$

converges in $L_{\varrho}^{\infty}\left(\mathbb{R}^{3}\right)$. 
5. The Ursell function. The Ursell function (relative to the origin) of our grand canonical ensemble with pair potential $u \in \mathscr{U}$ (see Section 3) is defined to be

$$
\omega_{\Lambda}(R)=\rho_{\Lambda}^{(2)}(R, 0)-\rho_{\Lambda}^{(1)}(R) \rho_{\Lambda}^{(1)}(0), \quad R \in \Lambda .
$$

For $z \in \mathcal{Z}$ the Ursell function can be expanded into an absolutely convergent power series

$$
\omega_{\Lambda}(R)=\sum_{N=2}^{\infty} a_{N, \Lambda}(R) z^{N}
$$

the coefficients of which depend on $u$ via the Mayer function $f$ defined in (2.4). They can be represented in the form

$$
a_{N, \Lambda}(R)=\frac{1}{(N-2) !} \int_{\Lambda^{N-2}} \sum_{\mathcal{C} \in \mathfrak{C}_{N}} \prod_{(i, j) \in \mathcal{C}} f\left(R_{i}-R_{j}\right) \mathrm{d} \boldsymbol{R}_{2, N},
$$

cf. Stell [15], where $R_{1}=R$ and $R_{2}=0, \mathfrak{C}_{N}$ is the set of connected graphs with $N$ vertices, labeled $1, \ldots, N$, and the product in $(5.3 \mathrm{a})$ runs over all bonds in $\mathcal{C}$ : the notation $(i, j)$ refers to a bond connecting vertices $i$ and $j$, where we use the convention that $i<j$ for $(i, j) \in \mathcal{C}$. For $N=2$ the representation (5.3a) is to be read as

$$
a_{2, \Lambda}(R)=f(R) .
$$

For our reference potential $u$ we consider a perturbation $v \in \mathscr{V}_{u}$ with $\|v\|_{\mathscr{V}_{u}} \leq$ $\delta_{0}(u)$, compare Section 2. For the complex pair potential $\widetilde{u}=u+\zeta v$ with $\zeta \in \mathbb{C},|\zeta| \leq$ 1 , we define $\widetilde{U}_{N}, V_{N}$, and $|V|_{N}$ as in (2.2), replacing $u$ by $\widetilde{u}, v$ and $|v|$, respectively, on the right-hand side. Using this notation it follows from (2.2) that $\widetilde{u}$ satisfies the stability bound

$$
\begin{aligned}
\operatorname{Re}\left(\widetilde{U}_{N}\left(\boldsymbol{R}_{N}\right)\right) & =U_{N}\left(\boldsymbol{R}_{N}\right)+\operatorname{Re}(\zeta) V_{N}\left(\boldsymbol{R}_{N}\right) \\
& \geq U_{N}\left(\boldsymbol{R}_{N}\right)-|V|_{N}\left(\boldsymbol{R}_{N}\right) \geq-N B
\end{aligned}
$$

for every coordinate vector $\boldsymbol{R}_{N} \in\left(\mathbb{R}^{3}\right)^{N}$, because $u-|v| \in \mathscr{U}$ by the definition of $\delta_{0}$. Furthermore, the associated (complex) Mayer function

$$
\widetilde{f}(R)=e^{-\beta \widetilde{u}(|R|)}-1, \quad R \in \mathbb{R}^{3},
$$

satisfies

$$
|\widetilde{f}(R)-f(R)| \leq \beta e^{-\beta(u-|v|)(|R|) \mid}|v(|R|)| \leq\left\{\begin{array}{cl}
\frac{1}{e\left(1-\delta_{0}\right)}\|v\|_{\mathscr{V}_{u}}, & |R|<r_{0}, \\
\frac{\beta e^{2 \beta B}}{\varrho(|R|)}\|v\|_{\mathscr{V}_{u}}, & |R| \geq r_{0} .
\end{array}\right.
$$

It follows that

$$
|\widetilde{f}(R)| \leq c_{\beta} w(R), \quad R \in \mathbb{R}^{3},
$$

where $c_{\beta}$ has been introduced in (2.5), and

$$
w(R)=\frac{1}{c_{\beta}}|f(R)|+C_{\beta} \frac{\delta_{0}}{\varrho(|R|)}
$$


for some suitably chosen constant $C_{\beta}>0$; note that $w$ is a positive function. Reducing the size of $\delta_{0}$, when necessary, we can make sure that

$$
q:=\int_{\mathbb{R}^{3}} w(R) \mathrm{d} R<1
$$

by virtue of (2.5). We fix $\delta_{0}=\delta_{0}(u)$ accordingly for the remainder of this paper. Note that (5.5) holds uniformly for all $\widetilde{u}=u+\zeta v$ with $\|v\|_{\mathscr{V}_{u}} \leq \delta_{0}$ and $\zeta \in \mathbb{C},|\zeta| \leq 1$.

As we have already mentioned in Section 4 the Mayer $f$-function belongs to the Banach space $L_{\varrho}^{\infty}\left(\mathbb{R}^{3}\right)$ introduced in (4.1), hence the function $w$ of (5.6) satisfies the assumptions of Proposition 4.2 and Corollary 4.3: As before we denote by $W_{n}, W_{\Sigma} \in L_{\varrho}^{\infty}\left(\mathbb{R}^{3}\right)$ the corresponding autoconvolutions (4.5) and their infinite series (4.11), respectively. Note that

$$
0<W_{n}(R) \leq W_{\Sigma}(R)
$$

for every $R \in \mathbb{R}^{3}$ and $n \in \mathbb{N}$, because $w$ is a positive function.

Now we fix a perturbation $v_{0} \in \mathscr{V}_{u}$ with $\left\|v_{0}\right\|_{\mathscr{V}_{u}} \leq \delta_{0}$, an activity parameter $z$ satisfying (3.1), and coordinates $R_{1}=R \in \Lambda$ and $R_{2}=0$. Let $N \geq 2$ and $\boldsymbol{R}_{2, N} \in \Lambda^{N-2}$ be further $N-2$ points in $\Lambda$. We use them to define entire functions

$$
f_{i j}(\zeta)=e^{-\beta\left(u\left(\left|R_{i}-R_{j}\right|\right)+\zeta v_{0}\left(\left|R_{i}-R_{j}\right|\right)\right)}-1, \quad 1 \leq i<j \leq N,
$$

of $\zeta \in \mathbb{C}$, and we observe that

$$
\varphi_{N}(\zeta)=\sum_{\mathcal{C} \in \mathfrak{C}_{N}} \prod_{(i, j) \in \mathcal{C}} f_{i j}(\zeta)
$$

is also an entire function, because the number of connected graphs with $N$ vertices is finite. For $0<\varepsilon \leq 1 / 2$ we can therefore apply Cauchy's integral formula to deduce that

$$
\left|\varphi_{N}(\varepsilon)-\varphi_{N}(0)\right|=\left|\frac{\varepsilon}{2 \pi \mathrm{i}} \int_{|\zeta|=1} \frac{\varphi_{N}(\zeta)}{\zeta(\zeta-\varepsilon)} \mathrm{d} \zeta\right| \leq \frac{\varepsilon}{\pi} \int_{0}^{2 \pi}\left|\varphi_{N}\left(e^{\mathrm{i} \theta}\right)\right| \mathrm{d} \theta
$$

and

$$
\begin{aligned}
\left|\varphi_{N}(\varepsilon)-\varphi_{N}(0)-\varepsilon \varphi_{N}^{\prime}(0)\right| \\
=\left|\frac{\varepsilon^{2}}{2 \pi \mathrm{i}} \int_{|\zeta|=1} \frac{\varphi_{N}(\zeta)}{\zeta^{2}(\zeta-\varepsilon)} \mathrm{d} \zeta\right| \leq \frac{\varepsilon^{2}}{\pi} \int_{0}^{2 \pi}\left|\varphi_{N}\left(e^{\mathrm{i} \theta}\right)\right| \mathrm{d} \theta .
\end{aligned}
$$

The absolute value of $\varphi_{N}$ can be estimated by means of a tree-graph inequality

$$
\left|\varphi_{N}(\zeta)\right| \leq e^{N \beta B} \sum_{\mathcal{T} \in \mathfrak{T}_{N}} \prod_{(i, j) \in \mathcal{T}}\left|f_{i j}(\zeta)\right|, \quad|\zeta| \leq 1
$$

where $\mathfrak{T}_{N}$ is the set of trees with $N$ vertices. Note that this inequality, which can be found in Ueltschi [16], makes use of the stability bound (5.4), which in turn requires $|\zeta| \leq 1$

By virtue of (5.5) we have the inequality

$$
\int_{\Lambda^{N-2}} \prod_{(i, j) \in \mathcal{T}}\left|f_{i j}(\zeta)\right| \mathrm{d} \boldsymbol{R}_{2, N} \leq \int_{\mathbb{R}^{N-2}} \prod_{(i, j) \in \mathcal{T}} c_{\beta} w\left(R_{i}-R_{j}\right) \mathrm{d} \boldsymbol{R}_{2, N}
$$


for any fixed tree $\mathcal{T} \in \mathfrak{T}_{N}$. Such a tree consists of (i) a "backbone" with, say, $n$ bonds and $n-1$ inner vertices, where $1 \leq n \leq N-1$, which connects the vertices 1 and 2, and (ii) $n+1$ subtrees rooted at all vertices of this backbone. One can first integrate (5.11) over all $N-n-1$ vertices of these subtrees besides their roots, with each of these integrals being bounded by $c_{\beta}$ according to (5.7); integrating over the inner vertices of the backbone thereafter constitutes an $n$-fold autoconvolution of $c_{\beta} w$, i.e.,

$$
\int_{\Lambda^{N-2}} \prod_{(i, j) \in \mathcal{T}}\left|f_{i j}(\zeta)\right| \mathrm{d} \boldsymbol{R}_{2, N} \leq c_{\beta}^{N-1} W_{n}\left(R_{1}-R_{2}\right)=c_{\beta}^{N-1} W_{n}(R) \leq c_{\beta}^{N-1} W_{\Sigma}(R),
$$

where we have used (5.8) for the final inequality. Note that this estimate is independent of the particular form of the tree $\mathcal{T}$. Therefore, making use of Cayley's result that $\mathfrak{T}_{N}$ consists of exactly $N^{N-2}$ different trees, we deduce from (5.10) the upper bound

$$
\int_{\Lambda^{N-2}}\left|\varphi_{N}(\zeta)\right| \mathrm{d} \boldsymbol{R}_{2, N} \leq e^{N \beta B} N^{N-2} c_{\beta}^{N-1} W_{\Sigma}(R), \quad|\zeta| \leq 1
$$

Using the functions $\varphi_{N}, N \geq 2$, we can extend (5.3) and (5.2) - given the fixed coordinate $R \in \Lambda$ - to scalar functions of a complex variable $\zeta$, namely

$$
\widehat{a}_{N, \Lambda}(\zeta)=\frac{1}{(N-2) !} \int_{\Lambda^{N-2}} \varphi_{N}(\zeta) \mathrm{d} \boldsymbol{R}_{2, N}
$$

and

$$
\widehat{\omega}_{\Lambda}(\zeta)=\sum_{N=2}^{\infty} \widehat{a}_{N, \Lambda}(\zeta) z^{N}
$$

For $\zeta=0$ we recover the original definitions (5.3) and (5.2). Since $\varphi_{N}$ is absolutely integrable with respect to $\boldsymbol{R}_{2, N} \in \Lambda^{N-2}$, cf. (5.12), and the integral is uniformly bounded for $|\zeta| \leq 1$, it follows that $\widehat{a}_{N, \Lambda}$ is also complex analytic for $|\zeta| \leq 1$. Furthermore, from $(5.9 \mathrm{~b})$ and $(5.12)$ it follows that

$$
\begin{aligned}
\left|\widehat{a}_{N, \Lambda}(\varepsilon)-\widehat{a}_{N, \Lambda}(0)-\varepsilon \widehat{a}_{N, \Lambda}^{\prime}(0)\right| & \leq 2 \varepsilon^{2} \frac{N^{N-2}}{(N-2) !} e^{N \beta B} c_{\beta}^{N-1} W_{\Sigma}(R) \\
& \leq \frac{2 \varepsilon^{2}}{c_{\beta}}\left(c_{\beta} e^{\beta B+1}\right)^{N} W_{\Sigma}(R)
\end{aligned}
$$

for $0<\varepsilon \leq 1 / 2$.

Since the infinite series (5.13) converges uniformly for $\zeta \in \mathbb{C},|\zeta| \leq 1$ (for the same fixed parameters $R, z$, and the same perturbation $v_{0} \in \mathscr{V}_{u}$ ), the complex extension $\widehat{\omega}$ of the Ursell function is also an analytic function of $\zeta$ in a neighborhood of the unit disk with

$$
\left|\widehat{\omega}_{\Lambda}(\varepsilon)-\widehat{\omega}_{\Lambda}(0)-\varepsilon \widehat{\omega}_{\Lambda}^{\prime}(0)\right| \leq \varepsilon^{2} z^{2} \frac{2 c_{\beta} e^{2(\beta B+1)}}{1-z c_{\beta} e^{\beta B+1}} W_{\Sigma}(R), \quad 0<\varepsilon \leq 1 / 2 .
$$

We already know from [4] that the original Ursell function (5.1) of $R \in \Lambda$ has a derivative $\partial \omega_{\Lambda} \in \mathscr{L}\left(\mathscr{V}_{u}, L^{\infty}(\Lambda)\right)$ with respect to $u$. Accordingly, (5.14) implies that when choosing $v_{0} \in \mathscr{V}_{u}, z$ as in (3.1), and $R \in \Lambda$ as above then

$$
\left(\left(\partial \omega_{\Lambda}\right) v_{0}\right)(R)=\widehat{\omega}_{\Lambda}^{\prime}(0)
$$


On the other hand, (5.14) is valid for every $z$ as in (3.1), $R \in \Lambda$, and independent of the particular choice of $v_{0} \in \mathscr{V}_{u}$ with $\left\|v_{0}\right\|_{\mathscr{V}_{u}} \leq \delta_{0}$. Therefore, denoting by $\omega_{\Lambda}$ and $\widetilde{\omega}_{\Lambda}$ the Ursell functions (5.1) associated with the reference potential $u$ and any perturbed potential $\widetilde{u}=u+v$ with $\|v\|_{\mathscr{V}_{u}} \leq \delta_{0} / 2$, we can rewrite (5.14) for

$$
v_{0}=\delta_{0} v /\|v\|_{\mathscr{V}_{u}} \quad \text { and } \quad \varepsilon=\|v\|_{\mathscr{V}_{u}} / \delta_{0}
$$

as

$$
\left|\left(\widetilde{\omega}_{\Lambda}-\omega_{\Lambda}-\left(\partial \omega_{\Lambda}\right) v\right)(R)\right| \leq z^{2} \frac{2}{\delta_{0}^{2}} \frac{c_{\beta} e^{2(\beta B+1)}}{1-z c_{\beta} e^{\beta B+1}}\|v\|_{\mathscr{V}_{u}}^{2} W_{\Sigma}(R),
$$

valid for every $R \in \Lambda$.

Starting from (5.13) with $\zeta=0$ or from (5.9a), respectively, the same line of argument leads to the corresponding estimates

$$
\left|\omega_{\Lambda}(R)\right| \leq z^{2} \frac{c_{\beta} e^{2(\beta B+1)}}{1-z c_{\beta} e^{\beta B+1}} W_{\Sigma}(R)
$$

and

$$
\left|\widetilde{\omega}_{\Lambda}(R)-\omega_{\Lambda}(R)\right| \leq z^{2} \frac{2}{\delta_{0}} \frac{c_{\beta} e^{2(\beta B+1)}}{1-z c_{\beta} e^{\beta B+1}}\|v\|_{\mathscr{V}_{u}} W_{\Sigma}(R)
$$

valid for every $R \in \Lambda$ and every $v \in \mathscr{V}_{u}$ with $\|v\|_{\mathscr{V}_{u}} \leq \delta_{0} / 2$.

Before we proceed we note that from (5.1) and the discussion in Section 3 one can readily conclude that

$$
\omega_{\Lambda}(R) \longrightarrow \omega(R):=\rho^{(2)}(R, 0)-\rho_{0}^{2}, \quad|\Lambda| \rightarrow \infty,
$$

uniformly on every compact subset of $R \in \mathbb{R}^{3}$ and for all activities $z$ in a compact subinterval of (3.1). We show next that this thermodynamical limit of the Ursell function belongs to the Banach space $L_{\varrho}^{\infty}\left(\mathbb{R}^{3}\right)$.

Proposition 5.1. Let $u \in \mathscr{U}$ and let $z$ satisfy (3.1), where $c_{\beta}$ and $B$ are given by (2.5) and (2.2), respectively. Then the thermodynamical limit of the Ursell function belongs to $L_{\varrho}^{\infty}\left(\mathbb{R}^{3}\right)$, i.e., there exists $c_{\omega}>0$ such that

$$
|\omega(R)| \leq c_{\omega}\left(1+|R|^{2}\right)^{-\alpha / 2}
$$

for every $R \in \mathbb{R}^{3}$.

Proof. We have mentioned already that the Mayer function $f$ belongs to $L_{\varrho}^{\infty}\left(\mathbb{R}^{3}\right)$ because of (2.1) so that the function $w$ defined in (5.6) satisfies the assumptions of Proposition 4.2 by virtue of (5.7). Accordingly, we deduce from Corollary 4.3 the existence of some constant $C>0$ such that

$$
W_{\Sigma}(R) \leq C\left(1+|R|^{2}\right)^{-\alpha} \quad \text { for } \quad R \in \mathbb{R}^{3} .
$$

For any fixed activity $z$ from the interval (3.1) we therefore obtain from (5.16a) the inequality

$$
\left|\omega_{\Lambda}(R)\right| \leq c_{\omega}\left(1+|R|^{2}\right)^{-\alpha}
$$


for some $c_{\omega}>0$ and all $R \in \Lambda$. Turning to the thermodynamical limit $|\Lambda| \rightarrow \infty$ the assertion thus follows from (5.17).

Some comments on Proposition 5.1 are in order.

The estimate (5.18) can be found in a paper by Groeneveld [2] with similar assumptions on the pair potential ${ }^{\S}$, but it appears that he only published a proof for nonnegative potentials (in [3]). On the other hand, Ruelle included in his book [13] a proof of the weaker statement that $\omega \in L^{1}\left(\mathbb{R}^{3}\right)$; see also [12].

A common way of estimating the decay of the Ursell function consists in rewriting the Mayer function in (5.10) as

$$
f(R)=\left(f(R) e^{a(R)}\right) e^{-a(R)}
$$

in such a way that $a$ satisfies a triangle inequality, and $f e^{a}$ is bounded and absolutely integrable. In this case the integral over the backbone considered above can be estimated by $e^{-a(R)}$ times an autoconvolution of $f e^{a}$, and this former factor $e^{-a(R)}$ provides an estimate for the rate of decay. In our case this approach could be realized with

$$
e^{a(R)}=|R|^{\alpha^{\prime}-3} \quad \text { for any } 3<\alpha^{\prime}<\alpha,
$$

but the resulting bound for the Ursell function is evidently suboptimal.

The bound (5.18), on the other hand, is optimal up to multiplicative constants, as follows from the cluster expansion (5.2), which gives

$$
\omega(R)=z^{2} f(R)+O\left(z^{3}\right), \quad z \rightarrow 0,
$$

according to (5.3b).

Now we finalize our investigation of the differentiability of the Ursell function.

TheOrem 5.2. Assume that $z$ satisfies (3.1). Then the thermodynamical limit of the Ursell function, considered a function of $u \in \mathscr{U}$, has a Fréchet derivative $\partial \omega \in \mathscr{L}\left(\mathscr{V}_{u}, L_{\varrho}^{\infty}\left(\mathbb{R}^{3}\right)\right)$. More precisely, if $\omega$ and $\widetilde{\omega}$ denote the thermodynamical limits of the Ursell functions corresponding to $u$ and $\widetilde{u}=u+v$, respectively, where $\|v\|_{\mathscr{V}_{u}} \leq$ $\delta_{0}(u) / 2$, then there exists $C_{\omega}=C_{\omega}(u, z)$, such that

$$
\begin{aligned}
\|\widetilde{\omega}-\omega\|_{L_{\varrho}^{\infty}\left(\mathbb{R}^{3}\right)} & \leq C_{\omega}\|v\|_{\mathscr{V}_{u}}, \\
\|\widetilde{\omega}-\omega-(\partial \omega) v\|_{L_{\varrho}^{\infty}\left(\mathbb{R}^{3}\right)} & \leq C_{\omega}\|v\|_{\mathscr{V}_{u}}^{2} .
\end{aligned}
$$

Proof. Let $u \in \mathscr{U}$ and $v \in \mathscr{V}_{u}$ satisfy $\|v\|_{\mathscr{V}_{u}} \leq \delta_{0}(u) / 2$. As in (5.15) we write $\widetilde{\omega}_{\Lambda}$ for the Ursell function associated with $\widetilde{u}=u+v$ and $\left(\partial \omega_{\Lambda}\right) v$ for the derivative of the Ursell function at $u$ in direction $v$. Then (5.20) readily follows from (5.15) and (5.16b) by using (5.19) and turning to the thermodynamical limit $|\Lambda| \rightarrow \infty$, compare (5.17).

6. Iterative Boltzmann inversion. Now we turn to the fixed point operator

$$
\Phi(u)=u+\gamma \log \frac{F(u)}{g^{\dagger}}
$$

\footnotetext{
$\S$ The notation in [2] concerning the assumptions on $u$ and the corresponding hypothesis is not fully clear, though.
} 
associated with the IBI method (1.2), where $\gamma>0$ is a fixed parameter. Recall that $F$ is the nonlinear operator (1.1) which takes a pair potential $u \in \mathscr{U}$ onto the corresponding radial distribution function $g$ in (3.2). This operator is associated with the corresponding grand canonical ensemble at fixed (inverse) temperature $\beta>0$ and fixed activity $z>0$, where for technical reasons we slightly restrict the admissible interval of the activity parameter, cf. (6.2) below. Note that Henderson [8] as well as Soper [14] considered the operator $F$ for a canonical ensemble at fixed temperature and fixed (counting) density $\rho_{0}$; it is possible to redo the subsequent analysis also for this case with $\rho_{0}$ sufficiently small by using the equivalence of ensembles and treating the activity as a function of the counting density and the potential, i.e., $z=z\left(\rho_{0}, u\right)$.

For fixed radial argument $r>0$ it is an immediate consequence of the results in [4] that the scalar function $u \mapsto(\Phi(u))(r)$ is differentiable with respect to $u$, and the corresponding derivative is given by

$$
\Phi^{\prime}(u) v=v+\gamma \frac{F^{\prime}(u) v}{F(u)}
$$

pointwise for $r>0$. However, since $F(u)$ - as a function of $r$ - decays exponentially near $r=0$ it is not at all obvious whether $\Phi^{\prime}$ actually is a Fréchet derivative in $\mathscr{L}\left(\mathscr{V}_{u}, \mathscr{V}_{u}\right)$. This will be established in Theorem 6.3 below. To prepare for this theorem we investigate the core region $0<r \leq r_{0}$ and the remaining interval $r>r_{0}$ separately. We start with the core region.

Lemma 6.1. Let $u \in \mathscr{U}$ and

$$
0<z \leq \bar{z}<\frac{1}{1+e} \frac{1}{c_{\beta} e^{2 \beta B+1}}
$$

be arbitrarily fixed. Then there exists $C>0$, depending on $u$ and on $\bar{z}$, but independent of $r \in\left(0, r_{0}\right]$, such that

$$
\begin{aligned}
|(\Phi(\widetilde{u})-\Phi(u))(r)| & \leq C\|\widetilde{u}-u\|_{\mathscr{V}_{u}} u(r), \\
\left|\left(\Phi(\widetilde{u})-\Phi(u)-\Phi^{\prime}(u)(\widetilde{u}-u)\right)(r)\right| & \leq C\|\widetilde{u}-u\|_{\mathscr{V}_{u}}^{2},
\end{aligned}
$$

uniformly for $\widetilde{u} \in \mathscr{U}$, provided that $\|\widetilde{u}-u\|_{\mathscr{V}_{u}}$ is sufficiently small.

Proof. Referring to the cavity distribution function $y$ defined in (3.3) we have

$$
F(u)=g=e^{-\beta u} y
$$

from which we deduce the representation

$$
F^{\prime}(u) v=-\beta e^{-\beta u} v y+e^{-\beta u}(\partial y) v,
$$

pointwise for $0<r \leq r_{0}$ and all $v \in \mathscr{V}_{u}$; here, $\partial y$ denotes the Fréchet derivative of $y$ with respect to $u$, compare Proposition 3.1.

Let $\widetilde{y}$ be the cavity distribution function associated with $\widetilde{u}=u+v$ for some $v \in \mathscr{V}_{u}$ sufficiently small. Then we have

$$
\begin{aligned}
\Phi(\widetilde{u})-\Phi(u) & =v+\gamma \log \frac{F(\widetilde{u})}{F(u)}=(1-\beta \gamma) v+\gamma \log \frac{e^{\beta \widetilde{u}} F(\widetilde{u})}{e^{\beta u} F(u)} \\
& =(1-\beta \gamma) v+\gamma \log (\widetilde{y} / y),
\end{aligned}
$$


and because $y$ is bounded from below, cf. Proposition 3.1, we can use the estimate

$$
|\log (1+x)-x| \leq 2 x^{2}, \quad|x|<1 / 2,
$$

to obtain

$$
\begin{aligned}
\Phi(\widetilde{u})-\Phi(u) & =(1-\beta \gamma) v+\gamma \frac{\widetilde{y}-y}{y}+O\left(\|v\|_{\mathscr{V}_{u}}^{2}\right) \\
& =(1-\beta \gamma) v+O\left(\|v\|_{\mathscr{V}_{u}}\right),
\end{aligned}
$$

uniformly for $0<r \leq r_{0}$ and $\|v\|_{\mathscr{V}_{u}}$ sufficiently small. Because of (2.1) and the definition (2.7) of $\|\cdot\|_{\mathscr{V}_{u}}$ this implies assertion (6.3a).

Starting from (6.6) and inserting the representations (6.1) and (6.4) of $\Phi^{\prime}(u)$ and $F^{\prime}(u)$, respectively, it follows that

$$
\begin{gathered}
\Phi(\widetilde{u})-\Phi(u)-\Phi^{\prime}(u) v=\gamma\left(\frac{\widetilde{y}-y}{y}-\frac{F^{\prime}(u) v}{F(u)}-\beta v\right)+O\left(\|v\|_{\mathscr{V}_{u}}^{2}\right) \\
=\frac{\gamma}{y}\left(\widetilde{y}-y-e^{\beta u} F^{\prime}(u) v-\beta v y\right)+O\left(\|v\|_{\mathscr{V}_{u}}^{2}\right) \\
=\frac{\gamma}{y}(\widetilde{y}-y-(\partial y) v)+O\left(\|v\|_{\mathscr{V}_{u}}^{2}\right)=O\left(\|v\|_{\mathscr{V}_{u}}^{2}\right)
\end{gathered}
$$

by virtue of Proposition 3.1, again, and this estimate also holds uniformly for $0<r \leq$ $r_{0}$. This proves assertion $(6.3 \mathrm{~b})$.

Lemma 6.2. Under the assumptions of Lemma 6.1 there exists $C>0$, such that

$$
\begin{aligned}
|(\Phi(\widetilde{u})-\Phi(u))(r)| & \leq C\|\widetilde{u}-u\|_{\mathscr{V}_{u}}\left(1+r^{2}\right)^{-\alpha / 2}, \\
\left|\left(\Phi(\widetilde{u})-\Phi(u)-\Phi^{\prime}(u)(\widetilde{u}-u)\right)(r)\right| & \leq C\|\widetilde{u}-u\|_{\mathscr{V}_{u}}^{2}\left(1+r^{2}\right)^{-\alpha / 2},
\end{aligned}
$$

uniformly for $r \geq r_{0}$, provided that $\|\widetilde{u}-u\|_{\mathscr{V}_{u}}$ is sufficiently small.

Proof. Using (3.2) and (5.17) we readily obtain the representation

$$
(F(u))(r)=g(r)=1+\frac{1}{\rho_{0}^{2}} \omega(R), \quad r=|R| \geq 0,
$$

and therefore (5.18), (5.20a), and the differentiability of $\rho_{0}$ with respect to $u$ imply a local Lipschitz bound

$$
|(F(\widetilde{u})-F(u))(r)| \leq C_{F}\|\widetilde{u}-u\|_{\mathscr{V}_{u}}\left(1+r^{2}\right)^{-\alpha / 2}
$$

with some $C_{F}=C_{F}(u, \bar{z})>0$ for all $\|\widetilde{u}-u\|_{\mathscr{V}_{u}}$ sufficiently small and all $r \geq 0$. Moreover, for $r \geq 0$ and $R \in \mathbb{R}^{3}$ with $|R|=r$ we further deduce from (6.8) that

$$
\left(F^{\prime}(u) v\right)(r)=\partial\left(\frac{1}{\rho_{0}^{2}} \omega(R)\right) v
$$

where the right-hand side denotes the derivative of the scalar function $u \mapsto \omega(R) / \rho_{0}^{2}$ with respect to $u$ in direction $v \in \mathscr{V}_{u}$. Again, using the differentiability of $\rho_{0}=\rho_{0}(u)$, Proposition 5.1, and Theorem 5.2, we conclude that

$$
\left|\left(F(\widetilde{u})-F(u)-F^{\prime}(u) v\right)(r)\right| \leq C_{F}^{\prime}\|v\|_{\mathscr{V}_{u}}^{2}\left(1+r^{2}\right)^{-\alpha / 2},
$$


for some $C_{F}^{\prime}>0$, all $v=\widetilde{u}-u \in \mathscr{V}_{u}$ sufficiently small, and all $r \geq 0$.

Since $F(u)$ is uniformly bounded from below for the given value of the activity and all $r \geq r_{0}$ according to Proposition 3.1 and (2.1), (6.9) implies that the fraction $(F(\widetilde{u})-F(u)) / F(u)$ is bounded by $1 / 2$ in absolute value, say, for $\|v\|_{\mathscr{V}_{u}}$ sufficiently small and all $r \geq r_{0}$. Accordingly,

$$
|\Phi(\widetilde{u})-\Phi(u)| \leq|v|+\gamma\left|\log \frac{F(\widetilde{u})}{F(u)}\right|=|v|+\gamma \frac{|F(\widetilde{u})-F(u)|}{F(u)}+2 \gamma\left|\frac{F(\widetilde{u})-F(u)}{F(u)}\right|^{2}
$$

by virtue of (6.5). Using once again that $F(u)$ is bounded from below for the respective radii $r \geq r_{0},(6.9)$ and (2.7) imply the first assertion (6.7a).

Using (6.1) the same argument as before yields

$$
\begin{aligned}
\left|\Phi(\widetilde{u})-\Phi(u)-\Phi^{\prime}(u) v\right| & =\gamma\left|\log \frac{F(\widetilde{u})}{F(u)}-\frac{F^{\prime}(u) v}{F(u)}\right| \\
& \leq \gamma\left|\frac{F(\widetilde{u})-F(u)-F^{\prime}(u) v}{F(u)}\right|+2 \gamma\left|\frac{F(\widetilde{u})-F(u)}{F(u)}\right|^{2}
\end{aligned}
$$

for $\|v\|_{\mathscr{V}_{u}}$ sufficiently small and all $r \geq r_{0}$. The second assertion (6.7b) thus follows from (6.10) and (6.9).

From Lemma 6.1 and Lemma 6.2 we immediately conclude our main result.

TheOrem 6.3. Let $u \in \mathscr{U}$, and let $z$ satisfy (6.2). Then there exists $C_{\Phi}=$ $C_{\Phi}(u, \bar{z})>0$ such that

$$
\|\Phi(\widetilde{u})-\Phi(u)\|_{\mathscr{V}_{u}} \leq C_{\Phi}\|\widetilde{u}-u\|_{\mathscr{V}_{u}}
$$

for $\|\widetilde{u}-u\|_{\mathscr{V}_{u}}$ sufficiently small. Moreover, $\Phi$ is Fréchet differentiable with respect to $u$ with $\Phi^{\prime}(u) \in \mathscr{L}\left(\mathscr{V}_{u}, \mathscr{V}_{u}\right)$, and

$$
\left\|\Phi(\widetilde{u})-\Phi(u)-\Phi^{\prime}(u)(\widetilde{u}-u)\right\|_{\mathscr{V}_{u}} \leq C_{\Phi}\|\widetilde{u}-u\|_{\mathscr{V}_{u}}^{2}
$$

for $\|\widetilde{u}-u\|_{\mathscr{V}_{u}}$ sufficiently small.

REMARK 6.4. We mention that for the particular choice $\gamma=1 / \beta$ of the relaxation parameter in IBI, compare (1.3), the first term on the right-hand side of (6.6) cancels, and hence, in this particular case we have the stronger Lipschitz bounds

$$
\begin{aligned}
\|\Phi(\widetilde{u})-\Phi(u)\|_{L_{\varrho}^{\infty}\left(\mathbb{R}^{3}\right)} & \leq C_{\Phi}\|\widetilde{u}-u\|_{\mathscr{V}_{u}}, \\
\left\|\Phi(\widetilde{u})-\Phi(u)-\Phi^{\prime}(u)(\widetilde{u}-u)\right\|_{L_{\varrho}^{\infty}\left(\mathbb{R}^{3}\right)} & \leq C_{\Phi}\|\widetilde{u}-u\|_{\mathscr{V}_{u}}^{2},
\end{aligned}
$$

under the same assumptions as in Theorem 6.3.

We now explain how this theorem provides a rigorous justification of IBI. To this end we assume that the given data $g^{\dagger}$ in (1.2) is the true radial distribution function associated with a pair potential $u^{\dagger} \in \mathscr{U}$, i.e.,

$$
g^{\dagger}=F\left(u^{\dagger}\right)
$$

and that $z$ satisfies (6.2). Further, let $u_{0} \in \mathscr{U}$ be an initial guess with $\left\|u_{0}-u^{\dagger}\right\|_{\mathscr{V}_{u^{\dagger}}}$ sufficiently small. Then it follows from Theorem 6.3 that the first iterate of IBI,

$$
u_{1}=u_{0}+\gamma \log \frac{F\left(u_{0}\right)}{g^{\dagger}}
$$


belongs to $\mathscr{U}$ again, because

$$
\left\|u_{1}-u^{\dagger}\right\|_{\mathscr{V}_{u^{\dagger}}}=\left\|\Phi\left(u_{0}\right)-\Phi\left(u^{\dagger}\right)\right\|_{\mathscr{V}_{u^{\dagger}}} \leq C_{\Phi}\left\|u_{0}-u^{\dagger}\right\|_{\mathscr{V}_{u^{\dagger}}} .
$$

Accordingly, one can continue iterating and determine further iterates $u_{2}, u_{3}, \ldots$ It stays an open problem, however, whether all iterates will stay within $\mathscr{U}$, or even converge to $u^{\dagger}$, eventually.

In practice IBI is usually applied with the potential of mean force as initial guess. Assuming as before that $u^{\dagger}$ is a Lennard-Jones type pair potential and that $g=g^{\dagger}$ of (6.11) is given exactly, then this amounts to choosing

$$
u_{0}=-\frac{1}{\beta} \log g^{\dagger} .
$$

Since the corresponding cavity distribution function $y^{\dagger}=e^{\beta u^{\dagger}} g^{\dagger}$ is bounded and strictly positive according to Proposition 3.1 we have

$$
-\frac{1}{\beta} \log g^{\dagger}(r)=u^{\dagger}-\frac{1}{\beta} \log y^{\dagger}=u^{\dagger}+O(1),
$$

uniformly for $0<r \leq r_{0}$. On the other hand it follows from Proposition 5.1 that there exists $c_{g}>0$ with

$$
\left|g^{\dagger}(r)-1\right| \leq c_{g}\left(1+r^{2}\right)^{-\alpha / 2}
$$

which implies

$$
\left|\log g^{\dagger}(r)\right| \leq C\left(1+r^{2}\right)^{-\alpha / 2}
$$

by virtue of (6.5) for some $C>0$ and $r$ sufficiently large. Moreover, since $y^{\dagger}$ has strictly positive lower and upper bounds it follows from the representation $g^{\dagger}=e^{-\beta u^{\dagger}} y^{\dagger}$ of the radial distribution function that (6.12b) extends to all $r \geq r_{0}$ after increasing $C$ appropriately, when necessary.

We thus conclude from (6.12) that the potential of mean force is a Lennard-Jones type pair potential with the same parameter $\alpha$, and therefore the first iteration of IBI is well-defined for this initial guess.

Acknowledgements. The results of this paper have first been presented at the Oberwolfach Mini-Workshop Cluster Expansions: From Combinatorics to Analysis through Probability (February 2017). The author is indebted to Roberto Fernández, Sabine Jansen, and Dimitrios Tsagkarogiannis for the invitation and the opportunity to contribute this presentation. During the workshop David C. Brydges, Aldo Procacci, and Daniel Ueltschi provided arguments which have considerably simplified our original proof of Theorem 5.2. This input and many discussions with further participants of this workshop are gratefully acknowledged.

\section{REFERENCES}

1. M.E. Fisher And D. Ruelle, The stability of many-particle systems, J. Math. Phys. 7 (1966), pp. 260-270.

2. J. Groeneveld, Rigorous bounds for the equation of state and pair correlation function of classical many-particle systems, in T.A. Bak (ed.), Statistical Mechanics. Foundations and Applications, pp. 110-145, Benjamin, New York (1967) 
3. J. Groeneveld, Estimation methods for Mayer's graphical expansions, in F. Harary (ed.), Graph Theory and Theoretical Physics, pp. 229-259, Academic Press, New York (1967)

4. M. HANKe, Fréchet differentiability of molecular distribution functions I. $L^{\infty}$ analysis, arXiv:1603.03899 [math-ph] (2016), Lett. Math. Phys., to appear.

5. M. Hanke, Fréchet differentiability of molecular distribution functions II. The Ursell function, arXiv:1603.03900 [math-ph] (2016), Lett. Math. Phys., to appear.

6. J.-P. Hansen and I.R. McDonald, Theory of Simple Liquids, Academic Press, Oxford, Fourth ed. (2013)

7. F. Harary (ed.), A Seminar on Graph Theory, Holt, Rinehart, and Winston, New York, 1967.

8. R.L. Henderson, A uniqueness theorem for fluid pair correlation functions, Phys. Lett. A 49 (1974), pp. 197-198.

9. L. Koralov, An inverse problem for Gibbs fields with hard core potential, J. Math. Phys. 48 (2007), 053301.

10. T. Kuna, J.L. Lebowitz, and E.R. Speer, Realizability of point processes, J. Stat. Phys. 129 (2007), 417-439.

11. V. Rühle, C. Junghans, A. Lukyanov, K. Kremer, and D. Andrienko, Versatile objectoriented toolkit for coarse-graining applications, J. Chem. Theory Comput. 5, 3211-3223 (2009)

12. D. Ruelle, Cluster property of the correlation functions of classical gases, Rev. Mod. Phys. 36, 580-584 (1964)

13. D. Ruelle, Statistical Mechanics: Rigorous Results, W.A. Benjamin Publ., New York (1969)

14. A.K. Soper, Empirical potential Monte Carlo simulation of fluid structure, Chemical Physics 202 (1996), pp. 295-306.

15. G. Stell, Cluster expansions for classical systems in equilibrium, in H.L. Fritsch and J.L. Lebowitz (eds.), The Equilibrium Theory of Classical Fluids, pp. II-171-II-266, Benjamin, New York (1964)

16. D. Ueltschi, An improved tree-graph bound, Oberwolfach Report No. 8/2017, DOI 10.4171/OWR/2017/8 